\title{
A Kinetic Study of the Growth of Aspergillus nidulans and Other Fungi
}

\author{
By A. P. J. TRINCI \\ Department of Microbiology, Queen Elizabeth College, \\ University of London, Campden Hill, London, W.8
}

(Accepted for publication I March 1969)

\begin{abstract}
SUMMARY
The growth kinetics of Aspergillus nidulans, Mucor hiemalis and Penicillium chrysogenum on solid media and in submerged culture were studied. Growth of $\boldsymbol{A}$. nidulans colonies on solid medium can be divided into the four phases of lag, exponential, deceleration and constant growth rate. The growth kinetics of $\boldsymbol{A}$. nidulans in submerged culture were similar to those commonly found for unicellular micro-organisms. The effects on colony radial growth rate, of glucose concentration, medium depth, oxygen partial pressure and temperature were studied. The radial growth rate of glucose-limited colonies of $A$. nidulans increased linearly with the log. of the initial glucose concentration. The effects of glucose concentration on internode length, hyphal density and hyphal diameter were also studied. At glucose concentrations above $1 \%(w / v)$ there was an inverse relation between the radial growth rate of A. nidulans colonies and their peripheral hyphal density. The relations between colony radial growth rate $\left(K_{r}\right)$ and germ tube specific growth rate $\left(\alpha_{g}\right)$ on solid media and specific growth rate in submerged culture $\left(\alpha_{s}\right)$ were investigated. Direct proportionality between $K_{r}$ and $\alpha_{s}$ of $A$. nidulans was found by varying specific growth rate by temperature changes. The germ tubes of $A$. nidulans conidia grew exponentially at a rate which was about 2.3 times as great as the specific growth rate of the organism in submerged culture. The colony radial growth rates of the three moulds could not be used as a measure of their relative specific growth rates in submerged culture.
\end{abstract}

\section{INTRODUCTION}

Colony diameter and radial growth rate are frequently used as parameters of fungal growth in bioassays and physiological investigations. However, as pointed out by Pirt (1967), we lack detailed knowledge of the laws governing the response of microbial colonies to their environment.

When fungi are grown in submerged culture so that all nutrients are present in excess and growth inhibitors are not accumulated, the organism grows exponentially (Pirt \& Callow, 1960) according to the equation:

$$
d M / d t=\alpha M,
$$

where $M=$ mass of culture at time $t$ and $\alpha$ is a constant known as the 'specific growth rate'. Integration of this equation gives:

$$
\ln M-\ln M_{0}=\alpha t \text {, }
$$


where $\ln =$ natural logarithm and $M_{0}=$ concentration of organism at zero time. The specific growth rate $(\alpha)$ is a direct measure of the growth rate of an organism and may be calculated from the expression:

$$
\alpha=\ln 2 / t_{d},
$$

where $t_{d}$ is the doubling or mean effective generation time. Several workers have shown that filamentous fungi grow exponentially in submerged culture (Zalokar, 1959a; Pirt \& Callow, 1960; Borrow et al. 1964). Swanson \& Stock (1966), however, reported that the growth of Microsporum quinckeanum in submerged culture was not logarithmic. They suggested that filamentous fungi do not grow exponentially because individual hyphae grow at a constant rate. Mandels (1965), in a review of the 'Kinetics of fungal growth', supports this view, stating that '... growth is not autocatalytic in filamentous fungi'. However, although unbranched hyphae grow at a constant rate (Trinci \& Banbury, 1967) mycelial hyphae are able to grow exponentially in submerged culture because they produce new growing points (i.e. branches) at intervals.

Colony diameter and radial growth rate are often used by mycologists to determine the optimum temperature for fungal growth (Brancato \& Golding, 1953). This suggests that there may be a constant relationship between the colony growth rate of a fungus and its specific growth rate. No one, however, has determined whether such a relationship exists. Further, although it is well known from the work of Smith (1924) that spore germ tubes increase in length at an exponential rate, it is not known if the specific growth rates of germ tubes and of the organism in submerged culture are related. If a relationship exists, measurement of germ tube length might prove to be a convenient parameter of growth to use in bioassays and other work.

\section{METHODS}

Organisms. The organisms used were Aspergillus nidulans (BWB 224 [Glasgow] recombinant ve y; this strain has a velvet morphology and yellow conidia); Penicillium chrysogenum WIS 54-I 255 (obtained from Biochemistry Department, University of Wisconsin, U.S.A.) and Mucor hiemalis (-) (Queen Elizabeth College strain Z 14).

Medium. The composition of the medium (DAN) was (g./1.): D-glucose, IO; $\mathrm{KH}_{2} \mathrm{PO}_{4}, 8.9 ; \mathrm{Na}_{2} \mathrm{HPO}_{4} \mathrm{I}_{2} \mathrm{H}_{2} \mathrm{O}, 8.9 ; \mathrm{NaNO}_{3}$, 6; EDTA, 0.6; $\mathrm{MgSO}_{4} 7 \mathrm{H}_{2} \mathrm{O}, 0.25$; $\mathrm{CaCl}_{2}, 0.05 ; \mathrm{ZnSO}_{4} 7 \mathrm{H}_{2} \mathrm{O}, 0.2 ; \mathrm{MnSO}_{4} 4 \mathrm{H}_{2} \mathrm{O}, 0.02 ; \mathrm{CuSO}_{4} 5 \mathrm{H}_{2} \mathrm{O}, 0.005 ; \mathrm{FeSO}_{4} \mathrm{H}_{2} \mathrm{O}$, $0.1 ; \mathrm{Na}_{2} \mathrm{SO}_{4}, 0.5 ; \mathrm{NaMoO}_{4} 2 \mathrm{H}_{2} \mathrm{O}, 0.005$.

The basal medium was modified when Mucor hiemalis was grown by replacing $\mathrm{NaNO}_{3}$ with $6 \mathrm{~g}$. $\left(\mathrm{NH}_{4}\right)_{2} \mathrm{SO}_{4} / 1$., decreasing the concentration of the phosphates by half and adding $0.5 \mathrm{mg}$. thiamine hydrochloride/l. Analar grade chemicals were used.

The medium was prepared in the following manner. The phosphates were mixed at $\times 4$ final strength and adjusted to $\mathrm{pH} 6.5$ with the appropriate phosphate solution. Sodium nitrate was added to the phosphate buffer at $\times 4$ final strength. Glucose solutions were prepared at $\times 5$ final strength and the EDTA chelated trace elements at $\times 20$ final strength. Glucose solutions were autoclaved at $115^{\circ}$ for $15 \mathrm{~min}$., whilst the trace elements and phosphate + nitrate solutions were sterilized separately by autoclaving at $12 \mathrm{I}^{\circ}$ for $\mathrm{I} 5 \mathrm{~min}$.; $200 \mathrm{ml}$. of medium were prepared by mixing four solutions aseptically: $50 \mathrm{ml}$. of phosphate + nitrate, $40 \mathrm{ml}$. of glucose, $10 \mathrm{ml}$. of EDTA chelated trace elements and $100 \mathrm{ml}$. of water. For solid media $3 \mathrm{~g}$. of Oxoid Ionagar no. 2 was added to the water (final concentration $15 \mathrm{~g} . / 1$.). 
When the effect of glucose concentration on growth was studied, the glassware used to prepare the medium was soaked in $2 \%$ quadralene (Fisons, Loughborough, Leicestershire) for $\mathrm{I} \mathrm{hr}$, washed six times in tap water and rinsed in distilled water.

Preparation of agar plates. $20 \mathrm{ml}$. volumes of medium were pipetted into $9 \mathrm{~cm}$. sterile plastic Petri dishes which had been levelled on the tripod table described by Pirt (1967). The plates were dried at $37^{\circ}$ for about 10 min. in the inverted position with the lids removed. The depth of medium in a plate was determined by measuring a thin vertical section cut from the medium with a razor blade.

Preparation of inocula and inoculation. Inocula were prepared by washing spores from McCartney slopes with about Io $\mathrm{ml}$. of $0.1 \%$ Tween 80 . The spores were washed twice with Ringer solution and then resuspended in sterile distilled water. Inocula used in spore germination experiments were kept up to Io days at $4^{\circ}$, during which time there was no significant decrease in viability.

(b)

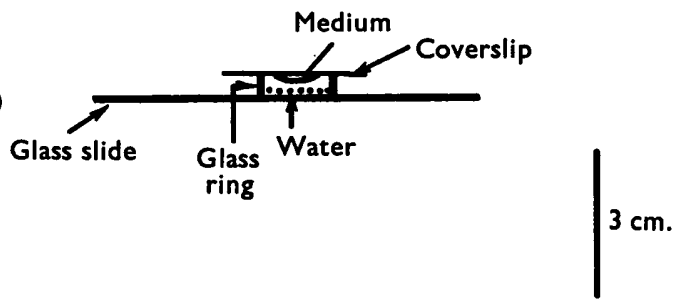

(a)

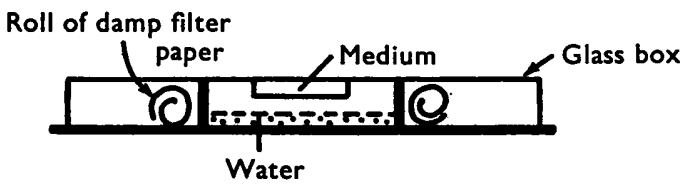

Fig. I. Various culture techniques: (a) culture chamber used to follow growth microscopically, $(b)$ slide culture used to follow growth microscopically.

Each plate was inoculated at three points with $\mathrm{I} \cdot 5$ to $2 \cdot 0 \mu \mathrm{l}$. of spore suspension dispensed from a repette (Pirt, 1967). The plates were incubated in biscuit tins with beakers of water containing rolls of filter paper. Unless stated otherwise, Aspergillus nidulans was grown at $37^{\circ}$ and Penicillium chrysogenum and Mucor hiemalis at $25^{\circ}$.

Measurements of spore and colony growth. Growth was recorded by measuring the diameter of colonies in two directions at right angles with a Shadowmaster (Pirt, 1967) and taking the mean of at least six replicates. Growth rates were calculated from measurements made at two intervals (9- to I8-hr period) whilst the colonies were growing at a constant rate.

The initial stages of colony growth and spore germination were followed by photographing the organism growing in a culture chamber or slide culture under a microscope (Fig. I $a, b$ ). Photographs were taken at 15 min. intervals with a Shackman $35 \mathrm{~mm}$. camera (Shackman \& Sons, Chesham, Bucks.). Measurements were made on the projected film (final magnification $\times 650$ or $\times 300$ ). Experiments were done in constant-temperature rooms.

Measurement of hyphal density and internode length. The cultures were fixed with formal + acetic acid + ethanol (FAA) and stained with trypan blue in lactophenol (Righelato, Trinci, Pirt \& Peat, 1968). The surface hyphae at the colony periphery were 
photographed with a Zeiss photomicroscope. Hyphal density was determined by projecting the negatives (final magnification $\times 200$ ) and counting the hyphae in focus crossing a $1000 \mu$ arc $500 \mu$ from the periphery of the colony. Each result was the mean of six replicates.

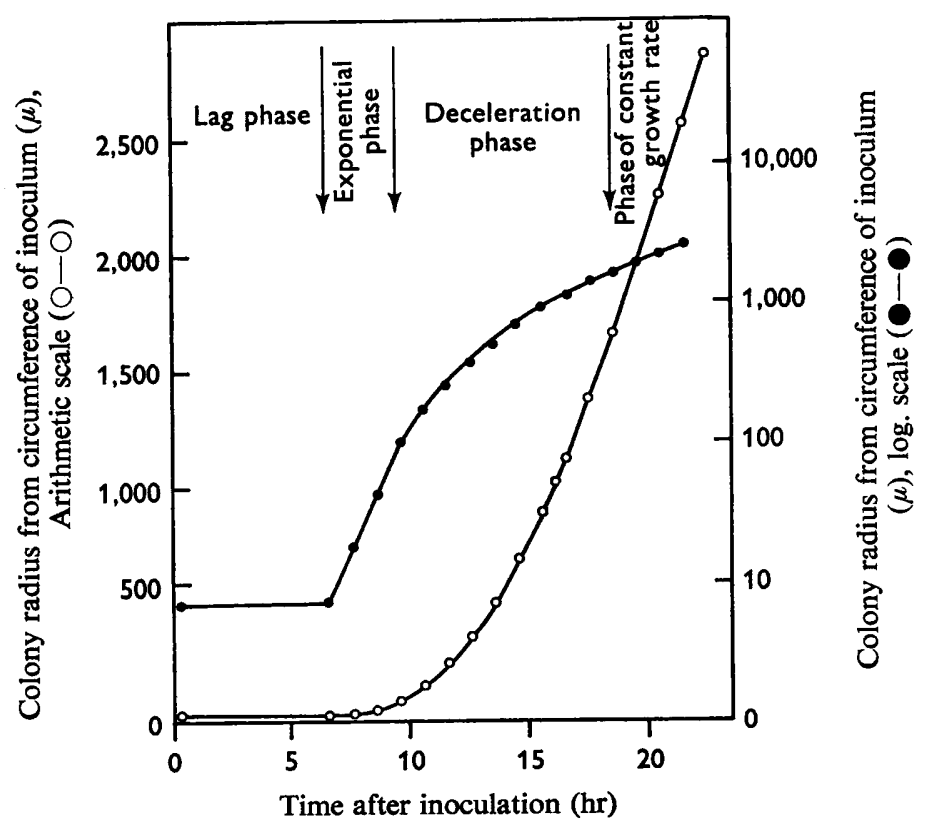

Fig. 2. Growth of surface colonies of Aspergillus nidulans.

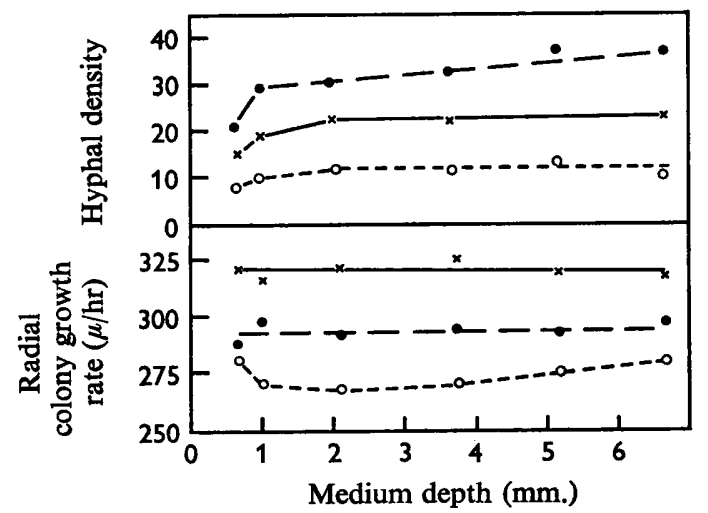

Fig. 3. Effect of medium depth on radial growth rate and hyphal density of Aspergillus nidulans colonies growing on $0.009 \mathrm{~g}$. glucose $/ 1 .\left(\mathrm{O}_{--} \mathrm{O}\right), 0.16 \mathrm{~g} . / 1 .(\times-\times)$ and I0.24 g./1. (0 - - - ).

Internode length of Mucor hiemalis was the mean of the first six internodes of main leading hyphae (growth is monopodial). Hyphal diameter was measured $200 \mu$ from the tips of main leading hyphae with a micrometer eyepiece. In both cases the results given are the mean of 6 hyphae per treatment. 
Growth of colonies at various oxygen partial pressures. Cultures in vented Petri dishes were placed in anaerobic culture jars which were then flushed out with the oxygen + nitrogen gas mixtures (gas flow $11 . / \mathrm{min}$.) for $5 \mathrm{~min}$. The anaerobic culture jars were lined with moist blotting paper and contained a beaker of $0.4 \%$ Pardee (1949) 'carbon dioxide buffer'.

Shake flask cultures. Submerged cultures were grown in $250 \mathrm{ml}$. conical flasks containing $25 \mathrm{ml}$. of medium. Each flask was inoculated with I $\mathrm{ml}$. of spore suspension (final density $\mathrm{I} \cdot 5$ to $2.5 \times 10^{6}$ spores $/ \mathrm{ml}$. of medium) and incubated on a Gallenkamp rotary shaker (A. Gallenkamp \& Co. Ltd., Christopher Street, London, E.C. 2) at $220 \mathrm{rev} . / \mathrm{min}$. Growth was followed by harvesting from flasks (at least 3/sample) with sintered glass crucibles (porosity X2). The mycelium was washed twice with $25 \mathrm{ml}$. of distilled water and dried overnight at $105^{\circ}$.

\section{RESULTS}

\section{Phases of colony growth}

Growth of Aspergillus nidulans colonies from the circumference of the inoculum was followed photographically (Fig. 2). Colony growth could be divided into four phases. After a lag phase the colony grew radially at an exponential rate (doubling time, $0.95 \mathrm{hr}$; specific growth rate, $0.73 \mathrm{hr}^{-1}$ ) until it was about $100 \mu$ from the circumference of the inoculum. The radial growth rate of the colony continued to increase during the third phase but no longer exponentially. This deceleration (from the previous exponential rate) phase lasted until the colony had grown about $\mathrm{I} \cdot \mathrm{I} \mathrm{mm}$. from the inoculum circumference. During the final growth phase the colony grew at a constant radial rate; this is the so-called 'linear phase of growth'. Quite different kinetics would be obtained if the inoculum centre had been taken as the origin for growth measurements. The kinetics of colony growth are identical with those of the growth of unbranched hyphae (Trinci \& Banbury, 1967).

\section{Effect of medium depth on growth rate and hyphal density}

Pirt (1967) found that medium depth had a significant influence on the growth rate of Escherichia coli colonies. To determine the effect of medium depth on the growth of Aspergillus nidulans colonies, plates were poured with different volumes of medium $(5,10,15,25,35$ and $45 \mathrm{ml}$., corresponding to depths of $0.66,0.99,2 \cdot 10,3 \cdot 74,5 \cdot 18$ and $6.68 \mathrm{~mm}$. respectively). The organism was grown on three different glucose concentrations and the radial growth rate and hyphal densities of the colonies were determined (Fig. 3). Medium depth had no influence on colony growth rate but there was a significant increase in hyphal density with increase in medium depth on all but the lowest glucose concentration.

\section{Colony growth into the medium}

Filamentous micro-organisms are able to grow down into an agar medium as well as over its surface. To investigate the extent of the submerged part of Aspergillus nidulans colonies, a thin vertical section of an FAA-fixed colony was cut with a razor blade $6.5 \mathrm{~mm}$. from its circumference. The section was stained with trypan blue in lactophenol, photographed along its length, and hyphal densities were determined at successive $0.5 \mathrm{~mm}$. intervals (Fig. 4). The results showed that the rate of growth down 
into the medium was apparently as fast as growth over the surface and that hyphal density decreased exponentially with depth below the surface of the colony. The colony was thus hemispherical in shape.

Effect of temperature on colony growth rate and germ tube specific growth rate on solid media and specific growth rate in submerged culture

Aspergillus nidulans was cultured at different temperatures to investigate the interrelationships between colony growth rate and germ-tube specific growth rate on solid media and specific growth rate in submerged culture.

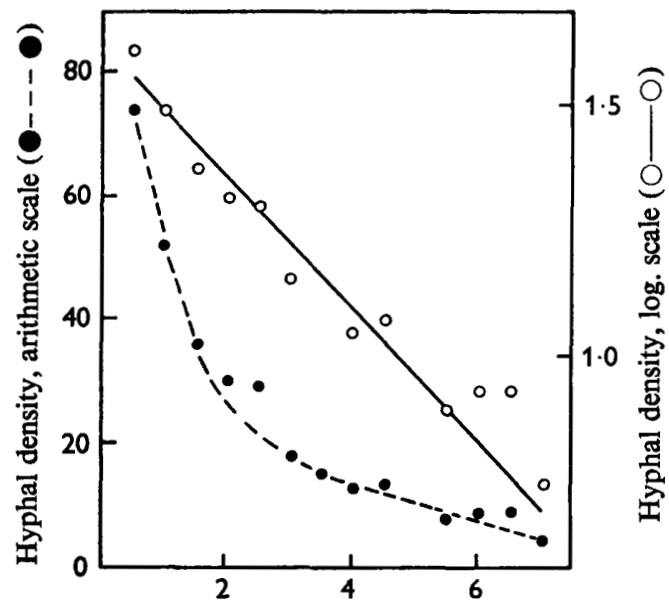

Depth below surface of medium (mm.)

Fig. 4

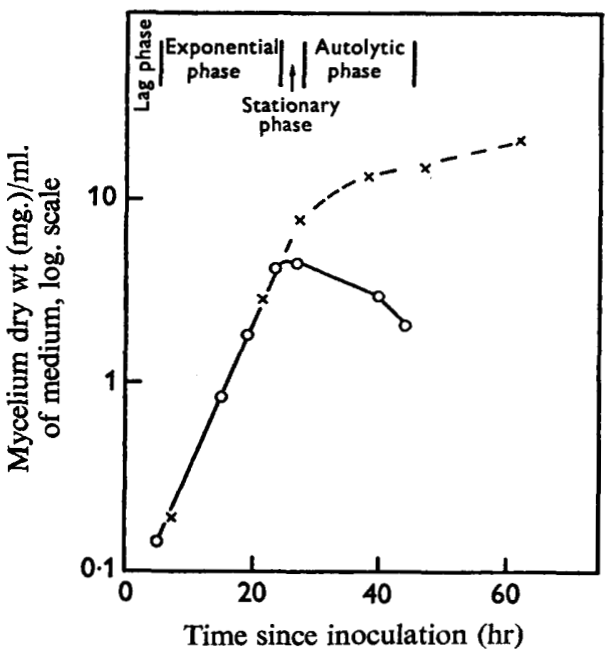

Fig. 5

Fig. 4. Change in hyphal density down a vertical section cut $6.5 \mathrm{~mm}$. from the circumference of an Aspergillus nidulans colony.

Fig. 5. Growth of Aspergillus nidulans in submerged culture on Io g. glucose/1. ( $-\longrightarrow)$ and $50 \mathrm{~g} . / \mathrm{l} .(\times---x)$.

\section{Specific growth rate in submerged culture}

Growth of Aspergillus nidulans in submerged culture at $30^{\circ}$ is shown in Fig. 5. Growth of the organism in a medium containing $10 \mathrm{~g}$. glucose/l. could be divided into lag, exponential, stationary and autolytic phases. Thus the growth kinetics of $A$. nidulans in submerged culture are similar to those commonly observed for unicellular micro-organisms. When $A$. nidulans was grown on a medium containing $50 \mathrm{~g}$.glucose/l. there was a decrease in growth rate when the organism concentration exceeded $7 \mathrm{mg}$./ml. of culture (Fig. 5). This decrease may have been caused either by the accumulation of toxic products in the medium or by growth becoming limited by the availability of oxygen; the $\mathrm{pH}$ of the medium did not drop below 6.3 . The concentration of carbon source in many mycological media exceeds $I \%(w / v)$ (it is $3 \%$ in Czapek Dox and $4 \%$ in Hesseltine medium for mucoraceous fungi), which, from these results, would seem to be too high for the maintenance of maximum specific growth rate in batch culture.

The specific growth rate of Aspergillus nidulans in submerged culture was determined over the temperature range $20^{\circ}$ to $37^{\circ}$ (Table I). 


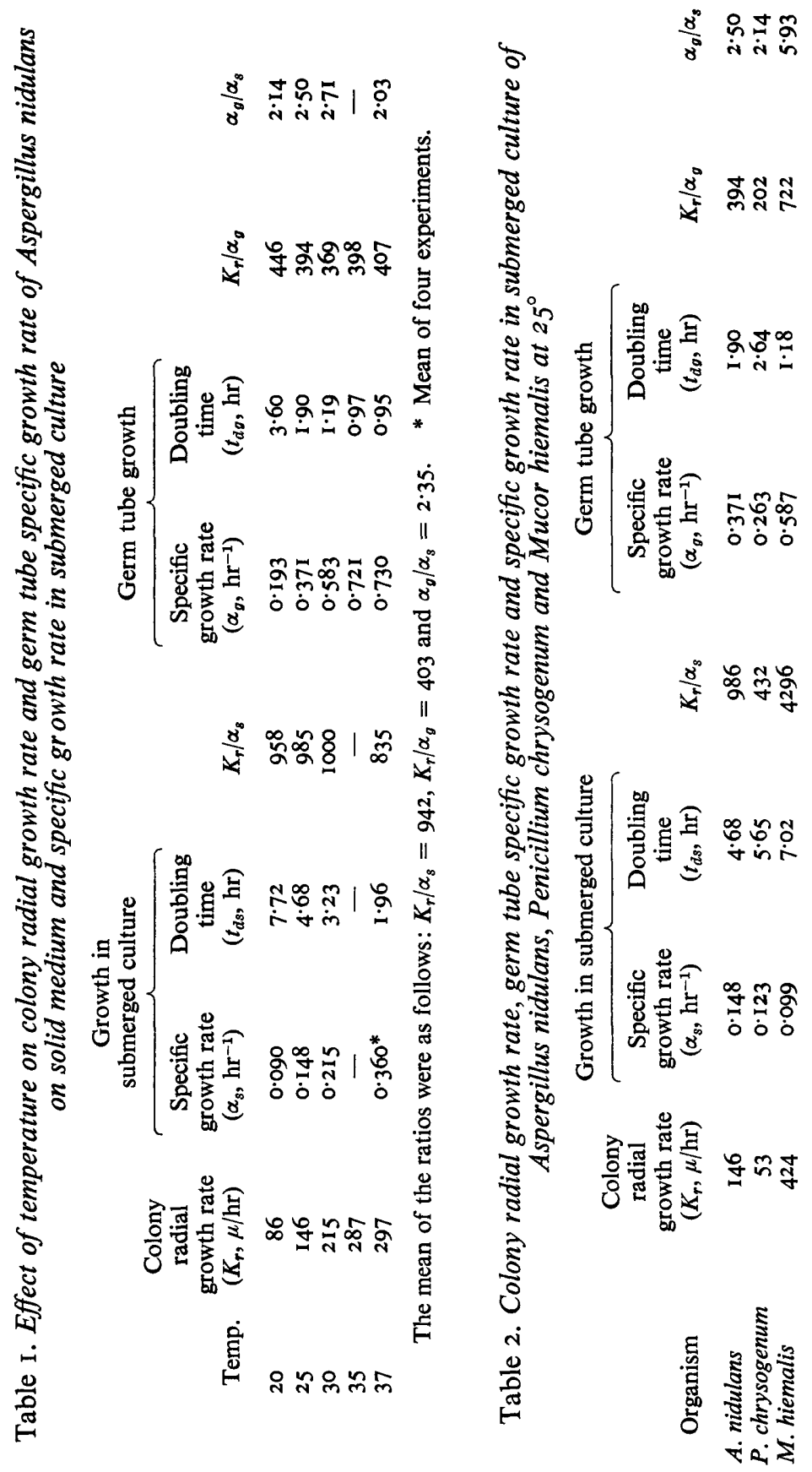




\section{Germ-tube specific growth rate}

Conidial germination was followed photographically in slide cultures (Fig. I c) until the germ tubes were about $150 \mu$ long. Growth of a germ tube at $37^{\circ}$ is shown in Fig. 6; the diameter of the conidium was included in all measurements and when the spore formed a second germ tube its length was also included. Conidia grew exponentially from the time when the germ tubes emerged until they were about $120 \mu$ long. Smith (1924) demonstrated the exponential nature of hyphal growth on solid medium but in his experiments measurements were only begun when the hyphae were about $230 \mu$ long. Plomley (1959) reported that for the first few hours after germination the germ tubes of Chaetomium conidia grew at a constant rate, not exponentially.

The mean doubling times $\left(t_{d g}\right)$ and specific growth rates $\left(\alpha_{g}, \mathrm{hr}^{-1}\right)$ of germ tubes were calculated over the temperature range $20^{\circ}$ to $37^{\circ}$ (Table I). When the conidia were not crowded together there was good agreement between the specific growth rates of germ tubes grown in different culture slides at the same temperature; e.g. at $37^{\circ}$ the germ tube doubling times ranged from 53 to $62 \mathrm{~min}$. with a mean of $57 \mathrm{~min}$.

\section{Colony radial growth rate}

The growth rate of Aspergillus nidulans colonies at various temperatures was also determined (Table I).

\section{Inter-relationships}

The results (Table 1 ) show that at least for part of the temperature range tested the changes in colony radial growth rate $\left(K_{r}\right)$ reflected changes in specific growth rate in submerged culture $\left(\alpha_{s}, \mathrm{hr}^{-1}\right)$. Between $20^{\circ}$ and $30^{\circ}$ the ratio, colony radial growth rate: specific growth rate in submerged culture $\left(K_{r} / \alpha_{s}\right)$ did not vary by more than $6.5 \%$ from the mean but at $37^{\circ}$ the ratio was higher than at other temperatures and there was a $12 \cdot 5 \%$ deviation from the mean. Pirt (1967), studying the growth of bacterial colonies, found a similar change in the ratio, colony growth rate: $\sqrt{ }($ specific growth rate), at high temperatures. He interpreted his results as indicating the presence of a temperature sensitive coefficient in colony growth. Rather surprisingly it was found that the germ tube specific growth rate $\left(\alpha_{g}, \mathrm{hr}^{-1}\right)$ was greater than the specific growth rate in submerged culture $\left(\alpha_{s}, \mathrm{hr}^{-1}\right)$ by a factor varying from about 2 to $2 \cdot 7$ (Table I). It was concluded that within experimental error the ratios, colony radial growth rate: specific growth rate in submerged culture $\left(K_{r} / \alpha_{s}\right)$, colony radial growth rate:germ tube specific growth rate $\left(K_{r} / \alpha_{g}\right)$ and germ tube specific growth rate: specific growth rate in submerged culture $\left(\alpha_{g} / \alpha_{s}\right)$ were constants.

Colony growth rates and germ tube specific growth rates on solid media and specific growth rates in submerged culture of Aspergillus nidulans, Penicillium chrysogenum and Mucor hiemalis at $25^{\circ}$

The colony radial growth rates, germ tube specific growth rates and specific growth rates in submerged culture of Aspergillus nidulans, Penicillium chrysogenum and Mucor hiemalis at $25^{\circ}$ were determined (Table 2). There was considerable variation between the three species in the ratio, colony radial growth rate:specific growth rate in submerged culture $\left(K_{r} / \alpha_{s}\right)$. Mucor hiemalis had the fastest colony growth rate but the slowest specific growth rate in submerged culture. Thus the growth rates of 
colonies of the three species cannot be taken as reliable indication of their relative specific growth rates in submerged culture. The rapid growth of $M$. hiemalis on plates is sustained by the transport of protoplasm synthesized within the colony to the comparatively few leading hyphae at the colony's periphery. The absence of septa may enable a greater region of the colony to contribute to its radial extension than might be the case in septate species. Certainly rapid protoplasmic streaming in hyphae in the direction of the colony's circumference is commonly observed in mucoraceous fungi. Thus, although an organism may only be able to synthesize protoplasm at a comparatively low rate (i.e. it has a low specific growth rate) it may be able to sustain a rapid radial rate of colony growth.

Influence of glucose concentration on colony growth rate, hyphal density, hyphal diameter and internode length

Pirt (1967) found that the relation between glucose concentration in the medium when it was the growth limiting nutrient and the initial radial growth rate $\left(K_{r}\right)$ of bacterial colonies could be represented by the formula:

$$
K_{r}=k_{1}\left(\sqrt{ } S_{0}-\sqrt{ } S_{i}\right),
$$

where $k_{1}$ was a constant, $S_{0}$ the initial glucose concentration and $S_{i}$ the glucose concentration which had to be exceeded before growth of the colony could occur; $S_{i}$ was termed the 'lag concentration' by Pirt. The following experiments were made to study the effect of glucose concentration on radial growth rate, hyphal density, hyphal diameter and internode length of fungal colonies.

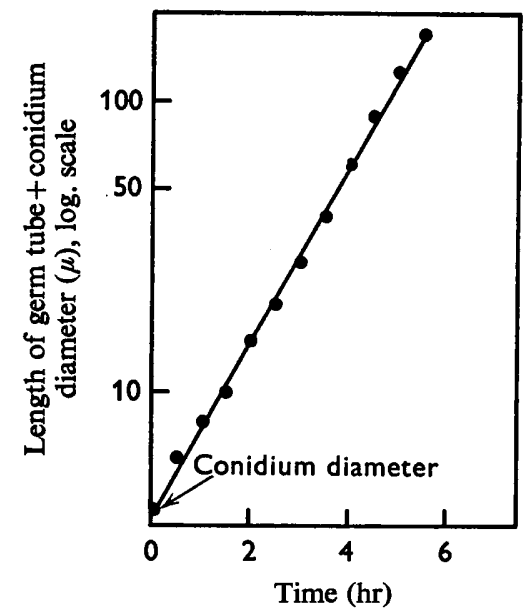

Fig. 6. Growth of an Aspergillus nidulans conidium.

The influence of glucose concentration on the growth of Aspergillus nidulans, Mucor hiemalis and Penicillium chrysogenum colonies was determined (Fig. 7 and 8). All three species grew rapidly even at the lowest glucose concentration (0.009 g./1.). At low glucose concentrations the growth of both $A$. nidulans and $M$. hiemalis colonies was glucose limited. The radial growth rate of $A$. nidulans colonies and the log. of the initial glucose concentration was linear over a considerable range (0.009 to $0 \cdot 15 \mathrm{~g}$./1.). The optimum glucose concentration for maximum colony growth rate was $0.075 \mathrm{~g} . / 1$. 
for $M$. hiemalis and $0.2 \mathrm{~g}$./1. for $A$. nidulans (over the lower range). These concentrations are much lower than the minimum glucose concentration required for bacterial colonies to attain their maximum rate of growth (e.g. $2.4 \mathrm{~g} . / 1$. for Streptococcus faecalis and $4^{\circ}$ g./1. for Escherichia coli; Pirt, 1967). At glucose concentrations greater than the optimum there was a decrease in the growth rate of $A$. nidulans and $M$. hiemalis colonies; in both species the colony radial growth rate declined linearly with the log. of the initial glucose concentration. At glucose concentrations above $10 \cdot 24 \mathrm{~g}$. $/ 1$. there was a second acceleration phase in the rate of growth of $A$. nidulans colonies.
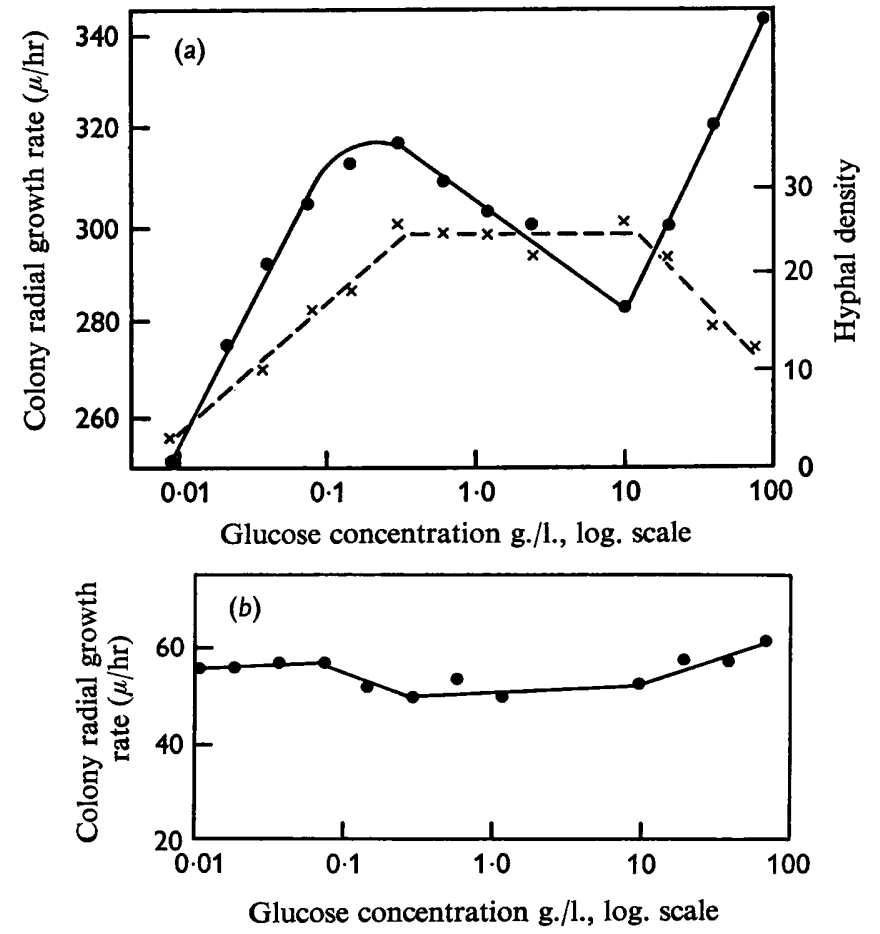

Fig. 7. (a) Effect of glucose concentration on the radial growth rate ( - -0$)$ and hyphal density $(x---x)$ of Aspergillus nidulans colonies. (b) Effect of glucose concentration on the radial growth rate of Penicillium chrysogenum colonies.

The peripheral hyphal density of Aspergillus nidulans colonies increased linearly with the log. of the initial glucose concentration between 0.009 and $0.3 \mathrm{~g}$. $/ 1$. and the decline in growth rate at higher concentrations was correlated with the attainment of the maximum hyphal density. The acceleration in growth rate of $A$. nidulans colonies which commenced at $\mathbf{2 0 . 4 8} \mathrm{g}$./1. was correlated with a decline in hyphal density; there was an inverse relationship between colony radial growth rate and hyphal density.

The mean internode length of the main leading hyphae (Fig. 8a) of Mucor hiemalis colonies was not influenced by glucose concentration between $0 \cdot 19$ and $8 \mathrm{I} \cdot 92 \mathrm{~g}$./1. but there was a significant decrease in internode length at $0.009 \mathrm{~g}$./1. Internode length is meaningless in $A$. nidulans since the hyphae of this organism branch apically as well as laterally (A. P. J. Trinci, unpublished observation). There was an increase in the mean diameter of the main leading hyphae of $M$. hiemalis colonies between 0.009 and 
I.2 g.glucose/1. (Fig. 8 b). At glucose concentrations above $\mathrm{I} \cdot 2 \mathrm{~g} . / 1$. hyphal diameter was apparently constant. The mean total length of the first six branches of $M$. hiemalis was also determined (Fig. $8 b$ ).

Growth of colonies of the slow-growing organism, Penicillium chrysogenum, was not glucose limited even at $0.009 \mathrm{~g}$./l.; growth of this organism must be limited by some other factor.

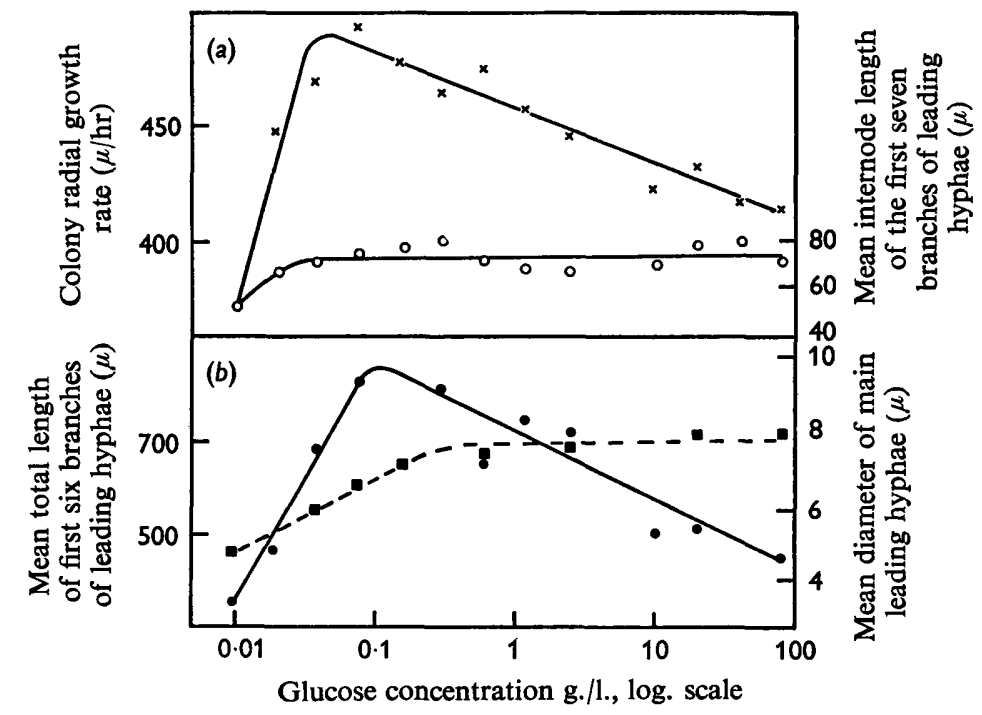

Fig. 8. (a) Effect of glucose concentration on the radial growth rate $(x-x)$ and hyphal internode length $(\mathrm{O}-\mathrm{O})$ of Mucor hiemalis colonies. (b) Effect of glucose concentration on the' total length of the first six branches of leading hyphae (-) and the diameter of leading hyphae ( - - - $\mathbf{-})$ of Mucor hiemalis colonies.

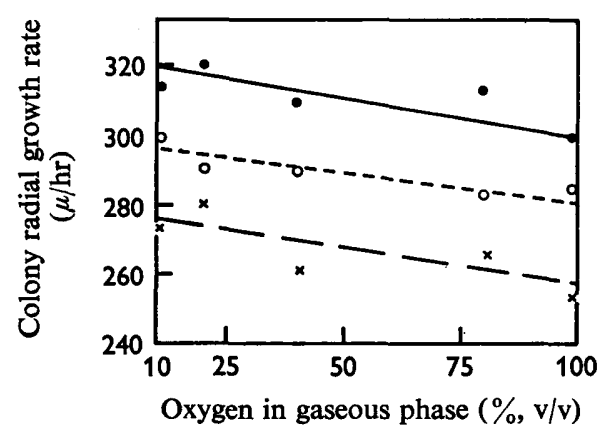

Fig. 9. Effect of oxygen concentration on the radial growth rate of Aspergillus nidulans colonies grown on $0.1 \mathrm{~g}$. glucose/1. $\left(\mathrm{O}_{--}\right)$, I0 g./1. $\left(x_{--} \times\right)$and $81 \cdot 92 \mathrm{~g} . / \mathrm{l}$. $(\bullet-\bullet)$

\section{Effect of glucose concentration on germ tube specific growth rate}

The specific growth rate (mean of 3 to 4 replicates) of Aspergillus nidulans germ tubes growing on media containing different glucose concentrations in culture chambers (Fig. I $a$ ) was determined (Table 3). There was little variation in the germ tube specific growth rate over the glucose range tested. Germ tube growth was probably 
not limited by external glucose concentration because the conidia had sufficient endogenous supplies of energy-containing compounds for germination. Thus germ tube specific growth rate probably cannot be used as a parameter of growth when studying the effect of nutrient concentration. However, the experiment demonstrates that high external glucose concentrations were not inhibitory to growth.

\section{Influence of oxygen partial pressure on colony growth rate}

Pirt (1967) found that at glucose concentrations of $2.5 \mathrm{~g} . / 1$. and above the radial rate of growth of Escherichia coli colonies was limited by oxygen availability. The inhibition of growth of Aspergillus nidulans colonies at glucose concentrations between 0.2 and 10.24 g./1. (Fig. 7a) may have been caused by oxygen becoming the growth limiting factor.

Table 3. Influence of glucose concentration on the doubling time and specific growth rate of germ tubes of Aspergillus nidulans

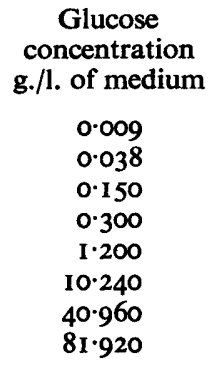

Mean doubling
time $\left(t_{d g}, \mathrm{hr}\right)$
$I \cdot 30$
$I \cdot I 0$
$I \cdot I 5$
$I \cdot 10$
$0 \cdot 98$
$I \cdot I 5$
$I \cdot I 2$
$I \cdot I 8$

Mean specific
growth rate $\left(\alpha_{g}, \mathrm{hr}^{-1}\right)$
$0.5331^{*}$
0.6300
0.6026
0.6300
0.7071
0.6026
0.6187
0.5873

* The germ tube specific growth rate was slightly lower in the culture chambers than in the culture slides (Fig. I).

Aspergillus nidulans colonies were grown in air for $24 \mathrm{hr}$, measured and then grown for a further $18 \mathrm{hr}$ at different oxygen partial pressures. At each glucose concentration growth was fastest at an oxygen concentration of $10 \%, \mathrm{v} / \mathrm{v}$ and slowest at $100 \%$ (Fig. 9); thus the growth rate of $A$. nidulans colonies is not oxygen limited even when grown on $8 \mathrm{r} \cdot 92 \mathrm{~g}$. glucose $/ 1$.

\section{DISCUSSION}

Growth of colonies of filamentous and unicellular micro-organisms differ in several respects. Initiation of the growth of bacterial colonies occurs only when the glucose concentration in the medium exceeds a certain value called the 'lag concentration' (Pirt, 1967); the lag concentration for Escherichia coli, Klebsiella aerogenes and Streptococcus faecalis was 0.090, 0.013 and $0.005 \mathrm{~g}$. glucose/1. respectively. However, all three fungal species in the present study grew rapidly on media containing 0.009 g. glucose/1. In addition fungal colonies attain their maximum radial growth rate at a significantly lower glucose concentration than bacterial colonies (e.g. $0.075 \mathrm{~g}$./1. for Mucor hiemalis as compared to $4^{\circ} \circ \mathrm{g}$./1. for $E$. coli). The filamentous habit permits variation in colony density such that the energy source available in the medium is used to maximum efficiency in extending the colony's diameter. The hyphal density control mechanism is presumably of significant selective advantage as it enables moulds to 
spread across substrates containing low concentrations of nutrients at near maximum radial growth rates; when a substrate containing a high concentration of nutrients is encountered, the colony grows more densely, produces aerial hyphae and may sporulate. Unicellular micro-organisms do not possess a specific mechanism to control the density of growth within the colony and typically form small colonies. A further advantage imparted by the filamentous habit is that it facilitates penetration of solid media and as a result the organism obtains nutrients which usually only become available to unicellular micro-organisms by diffusion. The radial growth rate of Aspergillus nidulans colonies, unlike Escherichia coli colonies, (Pirt, 1967) was never found to be limited by oxygen availability. Fungal colonies never attain the density of bacterial and yeast colonies at their periphery and it is probably because of this that the growth of leading hyphase does not become oxygen limited.

Internode length of Mucor hiemalis main hyphae was not influenced by glucose concentration between 0.0018 and $8.192 \%(\mathrm{w} / \mathrm{v})$. Lateral branch initiation is thus apparently not significantly influenced by nutrient concentration; differences in hyphal density result from variation in the number of branches which continue to grow once formed rather than by variation in the frequency of branch formation along main hyphae.

Colony radial growth rate increases linearly with the log. of the initial glucose concentration when this is the growth limiting nutrient. Gillie (1968) found that the rate of extension of Neurospora crassa colonies was proportional to the log. of the arginine concentration over the lower range, and it would thus seem that this is a general relationship for any growth limiting nutrient.

Synthesis of protoplasm contributing to radial extension of a colony is not confined to the tips of leading hyphae; Zalokar (1959b) showed that in Neurospora crassa portions of hyphae as far removed as $12 \mathrm{~mm}$. from the periphery of a colony contributed to the growth of its leading hyphae. If the environmental conditions within this peripheral growth zone became unfavourable to growth, the radial growth rate of the colony would decrease. The finding that germ tube specific growth rate of Aspergillus nidulans was not influenced by glucose concentration is an indication that high glucose concentrations are not in themselves inhibitory to growth. Thus the reduction in radial growth rate of $A$. nidulans and Mucor hiemalis colonies when growth was not glucose limited is probably due to changes in the medium resulting from growth of the organism, i.e. the accumulation of toxic products or $\mathrm{pH}$ changes. The rate at which these changes occur would presumably be related to hyphal density. The rapid growth of $A$. nidulans colonies on media containing $8 \mathrm{I} \cdot 92 \mathrm{~g}$. glucose $/ 1$. was associated with a substantial decrease in hyphal density. The radial growth rates of A. nidulans colonies on media containing I and $5 \%$ glucose (w/v) were different (Fig. $7 a$ ), although the mould had the same maximum specific growth rate on these media in submerged culture (Fig. 5). Thus colony radial growth rate is not a reliable parameter of specific growth rate in submerged culture in studies where nutrient concentration is varied.

Changes in specific growth rate of A. nidulans in submerged culture brought about by temperature changes were reflected in colony radial growth rate at least over most of the range tested. Colony radial growth rate would thus seem to be a reliable parameter to use to determine the optimum temperature for growth of a mould. However, it is clear from the results obtained that colony radial growth rate is not a meaningful 
parameter of growth (i.e. of specific growth rate in submerged culture) when comparisons are made between species.

Germ tube specific growth rate may prove to be a useful parameter of growth to use in certain bioassays and physiological investigations in which the effect on growth of factors other than nutrient concentration are being studied. The technique is less time consuming and more economical of media than submerged culture.

I wish to thank Professor S. J. Pirt for his helpful criticism during the course of this work, and Marian Parr for technical assistance. I would also like to thank the Central Research Fund of London University for a grant towards the cost of the time-lapse photographic equipment.

\section{REFERENCES}

Brancato, F. P. \& Golding, N. S. (1953). The diameter of the mould colony as a reliable measure of growth. Mycologia $\mathbf{4 5}, \mathbf{8 4 8}$.

Borrow, A., Brown, S., Jeffreys, E. G., Lesseli, R. H. J., Lloyd, E. C., Lloyd, P. B., Rothwell, A., Rothwell, B. \& SwaIt, J. C. (1964). The kinetics of metabolism of Gibberella fujikuroi in stirred culture. Can. J. Microbiol. 1o, 407.

GILLIE, O. J. (1968). Observations on the tube method of measuring growth rate in Neurospora crassa. J. gen. Microbiol. 51, 185.

MANDELS, G. R (1965). Kinetics of fungal growth. In The Fungi, vol. 1, p. 599. London: Academic Press.

PARDEe, A. B. (1949). Measurement of oxygen uptake under controlled pressures of carbon dioxide J. biol. Chem. 179, 1085.

PIRT, S. J. (1967). A kinetic study of the mode of growth of surface colonies of bacteria and fungi. J. gen. Microbiol. 47, 181 .

PIRT, S. J. \& Callow, D. S. (1960). Studies on the growth of Penicillium chrysogenum in continuousflow culture with reference to penicillin production. J. appl. Bact. 23, 87.

Plomley, N. J. B. (1959). Formation of the colony in the fungus Chaetomium. Aust. J. Biol. Sci. 12, 53.

Righelato, R. C., Trinci, A. P. J., Pirt, S. J. \& Peat, A. (1968). The influence of maintenance energy and growth rate on the metabolic activity, morphology and conidiation of Penicillium chrysogenum. J. gen. Microbiol. 5o, 399.

SmItH, J. H. (1924). On the early growth rate of the individual fungus hypha. New Phytol. 23, 65 .

Swanson, R. \& Stock, J. T. (1966). Biochemical alterations of dermatophytes during growth. Appl. Microbiol. 14, 438.

Trinci, A. P. J. \& Banbury, G. H. (1967). A study of the growth of the tall conidiophores of Aspergillus giganteus. Trans. Br. mycol. Soc. 50, 525.

ZALOKAR, M. (1959a). Enzyme activity and cell differentiation in Neurospora. Am. J. Bot. 46, 555.

ZALOKAR, M. (I959b). Growth and differentiation of Neurospora hyphae. Am. J. Bot. 46, 602. 